\title{
Identification of susceptibility loci for thoracic ossification of the posterior longitudinal ligament by whole-genome sequencing
}

\author{
PENG WANG ${ }^{1}$, XIAO LIU ${ }^{1}$, BIN ZHU $^{2}$, YUNLONG MA ${ }^{1}$, LEI YONG ${ }^{1}$, ZE TENG $^{3}$, \\ YONGQIANG WANG ${ }^{1}, \mathrm{CHEN} \mathrm{LIANG}^{1}$, GUANPING HE ${ }^{1}$ and XIAOGUANG LIU ${ }^{1}$ \\ ${ }^{1}$ Department of Orthopedics; ${ }^{2}$ Center for Pain Medicine; ${ }^{3}$ Department of Radiology, \\ Peking University Third Hospital, Beijing 100191, P.R. China
}

Received September 28, 2017; Accepted November 22, 2017

DOI: $10.3892 / \mathrm{mmr} .2017 .8171$

\begin{abstract}
Ossification of the posterior longitudinal ligament (OPLL) is a myelopathy commonly observed in the cervical spine. By contrast, thoracic OPLL (T-OPLL) is rare but more severe. Previous studies have identified several polymorphisms in osteogenic genes that are associated with the occurrence and development of cervical OPLL. However, few genetic studies have evaluated T-OPLL. The present study aimed to identify the genetic factors for OPLL by performing whole-genome sequencing (WGS) in 30 unrelated northern Chinese Han patients with T-OPLL. Using bioinformatics analyses and damaging-variant prediction algorithms, two deleterious variants [c.1534G $>A(p$. Gly512Ser)/collagen, type VI, $\alpha 1$ (COL6A1)] and [c.2275C >A(p.Leu759Ile)/inteleukin-17 receptor C (IL17RC)] were identified in seven unrelated patients. These two mutations resulted in markedly increased gene expression levels in peripheral blood samples. To the best of our knowledge, this is the first report to describe the use of WGS analysis of T-OPLL in the northern Chinese Han population. The results revealed two novel potentially pathogenic mutations in patients with T-OPLL.
\end{abstract}

\section{Introduction}

Ossification of the posterior longitudinal ligament (OPLL) involves pathological heterotopic ossification of the paravertebral ligament. Chronic compression of the spinal cord and nerve root leads to spinal cord compression symptoms and radiculopathy in patients with OPLL. The severity of the symptoms is associated with the size and segmentation of the ossifying ligament, and $70 \%$ of OPLL occurs in the cervical

Correspondence to: Professor Xiaoguang Liu, Department of Orthopedics, Peking University Third Hospital, 49 North Garden Street, Beijing 100191, P.R. China

E-mail:xglius@vip.sina.com

Key words: thoracic, ossification of the posterior longitudinal ligament, whole-genome sequencing, genetics spine, whereas $15 \%$ occurs in the thoracic spine (1). Notably, as early as 1972 , researchers reported that thoracic OPLL (T-OPLL) can cause thoracic spinal stenosis (2). Additionally, OPLL is primarily found in individuals in northeast Asia; the prevalence of cervical OPLL in Japanese individuals is $1.9-4.3 \%$ (3), which is much higher than the prevalence in Caucasians (0.01-1.7\%) (4). Moreover, the prevalence is higher in men than in women $(2.7: 1)$, and the mean age of onset is more than 40 years (5). A recent study published the prevalence of T-OPLL in Japanese individuals is $1.6 \%$ (6).

Treatment approaches for T-OPLL are limited, and surgery is currently the only effective treatment. However, owing to the unique features of blood supply and anatomical structures associated with this disease, surgery for T-OPLL is complicated, and the risk is extremely high. It is also difficult to avoid the occurrence of postoperative paralysis and surgical complications. Therefore, many studies have focused on the elucidation of the pathogenesis of T-OPLL, which is believed to involve interactions between genetic and environmental factors. Recent advances in genetic laboratory technology and sequencing of the human genome have enabled more specific studies to determine gene mutations causing OPLL or predisposing individuals to developing this condition. In genetic research on the occurrence and development of OPLL, the elucidation of the intrinsic mechanism may help us to further understand the disease, to establish assessment tools for determining T-OPLL severity and disease probability indexes, to achieve early disease detection and diagnosis, and to explore other auxiliary treatments. Thus, such studies are critical for improving therapeutic strategies for patients with this disease.

The thoracic spine experiences less activity than the cervical spine; thus, this region of the spine is subjected to less local biological stress than the cervical spine, and factors that mainly affect local mechanical stress and spine degeneration in cervical OPLL have little effect on T-OPLL. Previous studies have suggested that multiple polymorphisms in osteogenesis-related genes are associated with the development and progression of cervical OPLL. Additionally, extensive linkage and association studies have identified many genes linked to OPLL susceptibility. In previous reports, more than 16 susceptibility genes/loci have been shown to be linked to OPLL susceptibility (4), including collagen, type VI, $\alpha 1$ (COL6A1) (7-9), collagen, type XII, a2 (COL11A2) (10), 
bone morphogenetic protein 2 (BMP2) (11), transforming growth factor (TGF)- $\beta 1$ (12), interleukin (IL)-1 $\beta$ (13), IL-15 receptor $\alpha$ (IL-15RA) $(14,15)$, and runt-related transcription factor 2 (RUNX2) (16). However, these findings have not been sufficiently reproducible, and no genetic studies have assessed the causes of T-OPLL.

Thus, in this study, we used whole-genome sequencing (WGS) with high-density single nucleotide polymorphism (SNP) data combined with a predictive deleterious effects algorithm to identify genes or loci associated with T-OPLL in the Han Chinese population.

\section{Materials and methods}

Disease criteria and patients. The study protocol was approved by the ethical committee for human subjects of the Peking University Third Hospital. Informed consent was provided by all participating individuals. A total of 30 unrelated northern Chinese Han T-OPLL patients with myelopathy and/or neurological dysfunction [14 men, (mean age, 51.71 \pm 6.38 years); 16 women (mean age, 52.63 \pm 5.97 years)] and 5 unrelated healthy controls (mean age, 52.20 \pm 1.30 years, use for analysis of susceptibility gene expression levels in peripheral blood) were enrolled in this study from February 2010 to July 2016. Diagnosis of T-OPLL was performed by specialists based on clinical symptoms and radiologic examinations (including CT and magnetic resonance imaging) of the thoracic spine. The appearance of OPLL observed in radiographs was classified into four subtypes: i) Segmental; ii) continuous; iii) mixed; and iv) local (17). Neurological status was evaluated by the Japanese Orthopedic Association (JOA) score for thoracic myelopathy (a total of 11 points). Individuals who had lumbar spondylolisthesis, ankylosing spondylitis, diffuse idiopathic skeletal hyperostosis, and disc herniation of the thoracic spines were excluded in this study and did not take any drugs known to affect bone or calcium metabolism.

WGS. Genomic DNA was extracted from peripheral blood leukocytes using a standard method. To discover genetic variations, we performed WGS on 30 unrelated northern Chinese Han patients. Quality genomic DNA from the 30 samples was fragmented using a Covaris ultrasonicator. By adjusting shearing parameters, DNA fragments from each sample were concentrated in 500-bp peaks. These fragments were purified, end blunted, 'A' tailed, and adaptor ligated. DNA templates with adapters were then selectively enriched using polymerase chain reaction (PCR) to obtain a sufficient amount for the DNA library. The concentration of DNA for the libraries was quantified using a bioanalyser (Agilent Technologies, Inc., Santa Clara, CA, USA) and qPCR. Each qualified DNA library was sequenced on an Illumina HiSeq Xten platform using paired-end reads according to the manufacturer's instructions. Sequencing-derived raw image files were processed by Illumina base calling Software with default parameters, and sequence data for each individual were generated as paired-end reads, defined as 'raw data'.

Bioinformatics analysis. Bioinformatics analysis began with sequencing data (raw data from the Illumina machine). First, clean data were produced by data filtering of raw data. All clean data for each sample were mapped to the human reference genome (GRCh37/HG19). Burrows-Wheeler Aligner $(18,19)$ software was used to do the alignment. To ensure accurate variant calling, we followed recommended best practices for variant analysis with the Genome Analysis Toolkit. Local realignment around InDels and base quality score recalibration were performed using GATK $(20,21)$, with duplicate reads removed by Picard tools. The sequencing depth and coverage for each individual were calculated based on the alignments. In addition, a strict data analysis QC system throughout the entire pipeline was built to guarantee high-quality sequencing data.

To decrease noise in the sequencing data, data filtering was carried out, including: i) removal of reads containing the sequencing adapter; ii) removal of reads with a low-quality base ratio (base quality less than or equal to 5) that was more than 50\%; and iii) removal of reads with an unknown base ('N' base) ratio that was more than $10 \%$. Statistical analysis of data and downstream bioinformatics analyses were performed on the filtered, high-quality data, referred to as the "clean data'. Variant frequency was compared with the 1000G SNP database (http://www.1000genomes.org/). Potential deleterious effects of identified sequence variants were assessed by various algorithms, such as SIFT (http://sift.jcvi.org/) (22), PolyPhen-2 (http://genetics.bwh.harvard.edu/pph2/) (23), MutationTaster (http://www.mutationtaster.org/) (24), and GERP++ (http://mendel.stanford.edu/SidowLab/downloads/gerp/) (25).

Confirmation of variants. Sanger DNA sequencing (ABI 3730XL; Applied Biosystems; Thermo Fisher Scientific, Inc., Waltham, MA, USA) was used to confirm the accuracy of variants identified by WGS. The PCR fragments were submitted for Sanger sequencing at the Beijing Genomics Institute. Details of the two studied SNPs and the primer sequences are listed in Table I. PCR was performed with $20 \mathrm{ng}$ genomic DNA per $15 \mu \mathrm{l}$ reaction mixture, containing $0.2 \mu \mathrm{M}$ of each primer, $200 \mu \mathrm{M}$ of deoxyribonucleotides, $50 \mathrm{mM} \mathrm{KCl}$, $10 \mathrm{mM}$ Tris $\mathrm{HCl}$ (pH 8.3), $1.5 \mathrm{mM} \mathrm{MgCl}_{2}$ and 0.5 units of Taq DNA polymerase in a DNA Gradient PCR machine (Bio-Rad Laboratories, Inc., Hercules, CA, USA). The thermocycling conditions were as follows: initial denaturation at $95^{\circ} \mathrm{C}$ for $10 \mathrm{~min}$; followed by 35 cycles of $95^{\circ} \mathrm{C}$ for $30 \mathrm{sec}$, annealing at an assay-specific temperature $\left(48\right.$ to $\left.65^{\circ} \mathrm{C}\right)$ for $45 \mathrm{sec}$, and elongation at $72^{\circ} \mathrm{C}$ for $45 \mathrm{sec}$; and a final terminal elongation step at $72^{\circ} \mathrm{C}$ for $5 \mathrm{~min}$. The PCR products were analyzed by direct sequencing using a BigDye Terminator v3.1 Cycle Sequencing kit (Thermo Fisher Scientific, Inc.) with POP-7 ${ }^{\mathrm{TM}}$ Polymer in a 3730XL DNA Analyzer with Sequencing Analysis Software version 5.2 (Thermo Fisher Scientific, Inc.).

Protein conservation analysis. CLUSTAL W (http://www. genome.jp/tools/clustalw/) was used to compare homologous protein sequences among multiple species to analyse the consistency of amino acid sequences, particularly mutation sites, in seven representative species.

Plasma COL6A1 and IL17RC ELISAs. Plasma collection and storage were carried out using standard methods. Plasma COL6A1 (cat. no. HG21134) and IL17RC (cat. no. HG1762) levels were quantified using commercially available ELISA 
Table I. Details of the two SNPs in COL6AI and IL17RC and their associated primers.

\begin{tabular}{lccc}
\hline Gene & SNP ID & Nucleotide substitution $(\mathrm{M} / \mathrm{m})$ & Primer sequence \\
\hline COL6A1 & rs201153092 & G/A & Forward 5'-TGAAAGGGTGAGTGTCCAA-3' \\
IL17RC & & Reverse 5'-GTGCCCAGTCCACTAAAGAG-3' \\
& rs 199772854 & & Forward 5'-CCCAACTGCCAGACTTCCT-3' \\
& & & Reverse 5'-GCCACAGCCTGCGTAAAA-3'
\end{tabular}

SNP, single nucleotide polymorphism; COL6A1, collagen, type VI, $\alpha 1$; IL, interleukin;

Table II. Summary of whole-genome sequencing data.

\begin{tabular}{|c|c|c|c|c|c|c|c|c|}
\hline Sample & Raw reads & Raw bases $(\mathrm{Mb})$ & Clean reads & Clean bases $(\mathrm{Mb})$ & $\begin{array}{c}\text { Clean data } \\
\text { rate }(\%)\end{array}$ & $\begin{array}{c}\text { Clean read } \\
\text { Q20 }(\%)\end{array}$ & $\begin{array}{c}\text { Clean read } \\
\text { Q30 }(\%)\end{array}$ & $\begin{array}{c}\mathrm{GC} \\
\text { content }(\%)\end{array}$ \\
\hline $\mathrm{P} 1$ & 806578038 & 120986.71 & 802018468 & 120302.77 & 99.43 & 98.5 & 96.46 & 40.85 \\
\hline $\mathrm{P} 2$ & 803614916 & 120542.24 & 800270858 & 120040.63 & 99.58 & 98.57 & 96.61 & 41.02 \\
\hline P3 & 1166765398 & 175014.81 & 1162305438 & 174345.82 & 99.62 & 98.58 & 96.64 & 41 \\
\hline P4 & 742579698 & 111386.95 & 738204906 & 110730.74 & 99.41 & 98.51 & 96.49 & 40.83 \\
\hline P5 & 737867244 & 110680.09 & 734411142 & 110161.67 & 99.53 & 98.52 & 96.61 & 41.23 \\
\hline P6 & 695402032 & 104310.3 & 691857040 & 103778.56 & 99.49 & 98.5 & 96.47 & 40.88 \\
\hline P7 & 724494036 & 108674.11 & 721418588 & 108212.79 & 99.58 & 98.49 & 96.41 & 40.94 \\
\hline P8 & 776034852 & 116405.23 & 773219638 & 115982.95 & 99.64 & 98.48 & 96.5 & 41.28 \\
\hline P9 & 759932006 & 113989.8 & 755805520 & 113370.83 & 99.46 & 98 & 96.46 & 40.92 \\
\hline P10 & 781101726 & 117165.26 & 777211156 & 116581.67 & 99.5 & 98.53 & 96.53 & 40.93 \\
\hline P11 & 828771876 & 124315.78 & 824405368 & 123660.81 & 99.47 & 98.57 & 96.66 & 41.3 \\
\hline P12 & 845882256 & 126882.34 & 842286794 & 126343.02 & 99.57 & 98.52 & 96.58 & 41.25 \\
\hline P13 & 865879474 & 129881.92 & 860936622 & 129140.49 & 99.43 & 98.59 & 96.72 & 41.37 \\
\hline P14 & 737673162 & 110650.97 & 734221322 & 110133.2 & 99.53 & 98.53 & 96.55 & 40.88 \\
\hline P15 & 740541398 & 111081.21 & 735208756 & 110281.31 & 99.28 & 98.52 & 96.45 & 40.92 \\
\hline P16 & 792275714 & 118841.36 & 788504808 & 118275.72 & 99.52 & 98.49 & 96.4 & 40.94 \\
\hline P17 & 752134540 & 112820.18 & 749032976 & 112354.95 & 99.59 & 98.47 & 96.35 & 40.96 \\
\hline P18 & 728158076 & 109223.71 & 724409280 & 108661.39 & 99.49 & 98.49 & 96.44 & 40.92 \\
\hline P19 & 792928182 & 118939.23 & 789057438 & 118358.62 & 99.51 & 98.5 & 96.42 & 40.95 \\
\hline P20 & 777804644 & 116670.7 & 774841888 & 116226.28 & 99.62 & 98.53 & 96.51 & 41 \\
\hline P21 & 833487852 & 125023.18 & 829696094 & 124454.41 & 99.55 & 98.47 & 96.34 & 40.98 \\
\hline P22 & 759919330 & 113987.9 & 756111904 & 113416.79 & 99.5 & 98.58 & 96.62 & 40.9 \\
\hline P23 & 728707168 & 109306.08 & 725408206 & 108811.23 & 99.55 & 98.57 & 96.6 & 40.86 \\
\hline P24 & 742897576 & 111434.64 & 739389748 & 110908.46 & 99.53 & 98.58 & 96.62 & 40.9 \\
\hline $\mathrm{P} 25$ & 1094810746 & 164221.61 & 1090602492 & 163590.37 & 99.62 & 97.39 & 95.8 & 41.17 \\
\hline P26 & 772278386 & 115841.76 & 769004376 & 115350.66 & 99.58 & 98.59 & 96.65 & 40.88 \\
\hline P27 & 752896392 & 112934.46 & 748911760 & 112336.76 & 99.47 & 98.56 & 96.62 & 41.18 \\
\hline P28 & 756830924 & 113524.64 & 752633012 & 112894.95 & 99.45 & 98.61 & 96.78 & 41.16 \\
\hline P29 & 840021700 & 126003.26 & 835148570 & 125272.29 & 99.42 & 98.62 & 96.8 & 41.13 \\
\hline P30 & 778453666 & 116768.05 & 774362180 & 116154.33 & 99.47 & 98.55 & 96.59 & 40.82 \\
\hline Average & 797224100 & 119583.62 & 793363211 & 119004.48 & 99.51 & 98.48 & 96.52 & 41.01 \\
\hline
\end{tabular}

kits (Trust Specialty Zeal, San Francisco, CA, USA). All samples were assayed according to the manufacturer's instructions and were run in duplicate. The optical density of each well was determined using a microplate reader at $450 \mathrm{~nm}$. No interference and no cross reactivity were expected based on the manufacturer's instructions.
Reverse transcription-quantitative PCR (RT-qPCR). Total RNA was purified from blood using the SK1321 RNA Blood Mini kit (Sangon Biotech Co., Ltd., Shanghai, China). An on-column DNase digest (Sangon Biotech Co., Ltd.) was performed before the clean-up step to eliminate residual genomic DNA. cDNA was synthesized from total RNA $(2 \mu \mathrm{g})$ 
Table III. Data quality control. Strict data quality control was performed across the whole analysis pipeline for the clean data, the mapping data and the variant calling $\mathrm{Y}$ pass.

\begin{tabular}{|c|c|c|c|c|c|c|c|c|c|}
\hline Samples & $\begin{array}{c}\text { Clean } \\
\text { read1 } \\
\text { Q20 }(\%)\end{array}$ & $\begin{array}{c}\text { Clean } \\
\text { read1 } \\
\text { Q30 }(\%)\end{array}$ & $\begin{array}{c}\mathrm{GC} \\
\text { content } \\
(\%)\end{array}$ & $\begin{array}{l}\text { Mapping } \\
\text { rate }(\%)\end{array}$ & $\begin{array}{c}\text { Duplicate } \\
\text { rate }(\%)\end{array}$ & $\begin{array}{c}\text { Mismatch } \\
\text { rate (\%) }\end{array}$ & $\begin{array}{c}\text { Average } \\
\text { sequencing } \\
\text { depth }(X)\end{array}$ & Coverage $(\%)$ & $\begin{array}{c}\text { Coverage } \\
\text { at least } \\
4 \mathrm{X}(\%)\end{array}$ \\
\hline $\mathrm{P} 1$ & $\mathrm{Y}(98.50)$ & $\mathrm{Y}(96.46)$ & $\mathrm{Y}(40.85)$ & $\mathrm{Y}(99.54)$ & $\mathrm{Y}(11.84)$ & $\mathrm{Y}(0.63)$ & $\mathrm{Y}(35.10)$ & $\mathrm{Y}(99.09)$ & $\mathrm{Y}(98.69)$ \\
\hline P2 & $\mathrm{Y}(98.57)$ & $\mathrm{Y}(96.61)$ & $\mathrm{Y}(41.02)$ & Y(99.63) & $Y(10.85)$ & $\mathrm{Y}(0.56)$ & $\mathrm{Y}(35.63)$ & Y(99.06) & $\mathrm{Y}(98.68)$ \\
\hline P3 & $\mathrm{Y}(98.58)$ & $\mathrm{Y}(96.64)$ & $\mathrm{Y}(41.00)$ & $\mathrm{Y}(99.72)$ & $Y(9.32)$ & $\mathrm{Y}(0.59)$ & $\mathrm{Y}(52.43)$ & $\mathrm{Y}(99.82)$ & $\mathrm{Y}(99.63)$ \\
\hline P4 & $\mathrm{Y}(98.51)$ & $\mathrm{Y}(96.49)$ & $\mathrm{Y}(40.83)$ & $\mathrm{Y}(99.55)$ & $\mathrm{Y}(11.78)$ & $\mathrm{Y}(0.63)$ & $\mathrm{Y}(32.39)$ & Y(99.08) & $\mathrm{Y}(98.66)$ \\
\hline P5 & $\mathrm{Y}(98.52)$ & $\mathrm{Y}(96.61)$ & $\mathrm{Y}(41.23)$ & $\mathrm{Y}(99.53)$ & $Y(10.67)$ & $\mathrm{Y}(0.56)$ & $\mathrm{Y}(32.64)$ & $\mathrm{Y}(99.79)$ & $\mathrm{Y}(99.41)$ \\
\hline P6 & $\mathrm{Y}(98.50)$ & $\mathrm{Y}(96.47)$ & $\mathrm{Y}(40.88)$ & $\mathrm{Y}(99.61)$ & $Y(10.75)$ & $\mathrm{Y}(0.60)$ & $\mathrm{Y}(30.78)$ & $\mathrm{Y}(99.07)$ & $\mathrm{Y}(98.60)$ \\
\hline P7 & $\mathrm{Y}(98.49)$ & $\mathrm{Y}(96.41)$ & $\mathrm{Y}(40.94)$ & $\mathrm{Y}(99.66)$ & $Y(11.05)$ & $\mathrm{Y}(0.58)$ & $\mathrm{Y}(32.06)$ & $\mathrm{Y}(99.04)$ & $\mathrm{Y}(98.61)$ \\
\hline P8 & Y(98.48) & Y(96.50) & $\mathrm{Y}(41.28)$ & Y(99.54) & $\mathrm{Y}(10.08)$ & $\mathrm{Y}(0.56)$ & $\mathrm{Y}(34.62)$ & Y(99.14) & $\mathrm{Y}(98.71)$ \\
\hline P9 & $\mathrm{Y}(98.00)$ & $\mathrm{Y}(96.46)$ & $\mathrm{Y}(40.92)$ & $\mathrm{Y}(99.60)$ & $\mathrm{Y}(11.48)$ & $\mathrm{Y}(0.61)$ & $\mathrm{Y}(33.34)$ & $\mathrm{Y}(99.07)$ & $\mathrm{Y}(98.67)$ \\
\hline P10 & $\mathrm{Y}(98.53)$ & $\mathrm{Y}(96.53)$ & $\mathrm{Y}(40.93)$ & $\mathrm{Y}(99.63)$ & $\mathrm{Y}(12.61)$ & $\mathrm{Y}(0.58)$ & $\mathrm{Y}(33.90)$ & $\mathrm{Y}(99.17)$ & $\mathrm{Y}(98.69)$ \\
\hline P11 & $\mathrm{Y}(98.57)$ & $\mathrm{Y}(96.66)$ & $\mathrm{Y}(41.30)$ & $\mathrm{Y}(99.55)$ & $\mathrm{Y}(12.50)$ & $\mathrm{Y}(0.54)$ & $\mathrm{Y}(35.89)$ & $\mathrm{Y}(99.80)$ & $\mathrm{Y}(99.48)$ \\
\hline $\mathrm{P} 12$ & $\mathrm{Y}(98.52)$ & $\mathrm{Y}(96.58)$ & $\mathrm{Y}(41.25)$ & $\mathrm{Y}(99.55)$ & $\mathrm{Y}(10.47)$ & $\mathrm{Y}(0.55)$ & $\mathrm{Y}(37.59)$ & $\mathrm{Y}(99.82)$ & $\mathrm{Y}(99.52)$ \\
\hline P13 & $\mathrm{Y}(98.59)$ & $\mathrm{Y}(96.72)$ & $\mathrm{Y}(41.37)$ & $\mathrm{Y}(99.57)$ & $\mathrm{Y}(13.70)$ & $\mathrm{Y}(0.53)$ & $\mathrm{Y}(36.96)$ & $\mathrm{Y}(99.12)$ & $\mathrm{Y}(98.76)$ \\
\hline P14 & $\mathrm{Y}(98.53)$ & $\mathrm{Y}(96.55)$ & $\mathrm{Y}(40.88)$ & $\mathrm{Y}(99.65)$ & $\mathrm{Y}(11.22)$ & $\mathrm{Y}(0.59)$ & $\mathrm{Y}(32.56)$ & $\mathrm{Y}(99.09)$ & $\mathrm{Y}(98.67)$ \\
\hline P15 & $\mathrm{Y}(98.52)$ & $\mathrm{Y}(96.45)$ & $\mathrm{Y}(40.92)$ & $\mathrm{Y}(99.63)$ & $\mathrm{Y}(10.90)$ & $\mathrm{Y}(0.55)$ & $\mathrm{Y}(32.68)$ & Y(99.09) & $\mathrm{Y}(98.66)$ \\
\hline P16 & $\mathrm{Y}(98.49)$ & $\mathrm{Y}(96.40)$ & $\mathrm{Y}(40.94)$ & $\mathrm{Y}(99.58)$ & $\mathrm{Y}(11.39)$ & $\mathrm{Y}(0.57)$ & $\mathrm{Y}(34.71)$ & $\mathrm{Y}(99.78)$ & $\mathrm{Y}(99.43)$ \\
\hline P17 & $\mathrm{Y}(98.47)$ & $\mathrm{Y}(96.35)$ & $\mathrm{Y}(40.96)$ & $\mathrm{Y}(99.57)$ & $\mathrm{Y}(10.13)$ & $\mathrm{Y}(0.56)$ & $\mathrm{Y}(33.54)$ & $\mathrm{Y}(99.76)$ & $\mathrm{Y}(99.40)$ \\
\hline P18 & $\mathrm{Y}(98.49)$ & $\mathrm{Y}(96.44)$ & $\mathrm{Y}(40.92)$ & $\mathrm{Y}(99.54)$ & $Y(10.86)$ & $\mathrm{Y}(0.64)$ & $\mathrm{Y}(32.12)$ & $\mathrm{Y}(99.09)$ & $\mathrm{Y}(98.66)$ \\
\hline P19 & $\mathrm{Y}(98.50)$ & $\mathrm{Y}(96.42)$ & $\mathrm{Y}(40.95)$ & $\mathrm{Y}(99.60)$ & $\mathrm{Y}(10.99)$ & $\mathrm{Y}(0.55)$ & $\mathrm{Y}(35.05)$ & $\mathrm{Y}(99.12)$ & $\mathrm{Y}(98.73)$ \\
\hline P20 & $\mathrm{Y}(98.53)$ & $\mathrm{Y}(96.51)$ & $\mathrm{Y}(41.00)$ & $\mathrm{Y}(99.47)$ & $\mathrm{Y}(9.63)$ & $\mathrm{Y}(0.57)$ & $\mathrm{Y}(34.80)$ & $\mathrm{Y}(99.80)$ & $\mathrm{Y}(99.46)$ \\
\hline P21 & $\mathrm{Y}(98.47)$ & $\mathrm{Y}(96.34)$ & $\mathrm{Y}(40.98)$ & $\mathrm{Y}(99.46)$ & $\mathrm{Y}(10.91)$ & $\mathrm{Y}(0.57)$ & $\mathrm{Y}(36.67)$ & $\mathrm{Y}(99.78)$ & $\mathrm{Y}(99.47)$ \\
\hline P22 & $\mathrm{Y}(98.58)$ & $\mathrm{Y}(96.62)$ & $\mathrm{Y}(40.90)$ & $\mathrm{Y}(99.52)$ & $\mathrm{Y}(10.27)$ & $\mathrm{Y}(0.54)$ & $\mathrm{Y}(33.78)$ & $\mathrm{Y}(99.11)$ & $\mathrm{Y}(98.69)$ \\
\hline P23 & $\mathrm{Y}(98.57)$ & $\mathrm{Y}(96.60)$ & $\mathrm{Y}(40.86)$ & $\mathrm{Y}(99.54)$ & $\mathrm{Y}(9.64)$ & $\mathrm{Y}(0.54)$ & $\mathrm{Y}(32.65)$ & $\mathrm{Y}(99.07)$ & $\mathrm{Y}(98.63)$ \\
\hline P24 & $\mathrm{Y}(98.58)$ & $\mathrm{Y}(96.62)$ & $\mathrm{Y}(40.90)$ & $\mathrm{Y}(99.53)$ & $\mathrm{Y}(10.30)$ & $\mathrm{Y}(0.54)$ & $\mathrm{Y}(33.10)$ & $\mathrm{Y}(99.05)$ & $\mathrm{Y}(98.63)$ \\
\hline $\mathrm{P} 25$ & $\mathrm{Y}(97.39)$ & $\mathrm{Y}(95.80)$ & $\mathrm{Y}(41.17)$ & $\mathrm{Y}(99.78)$ & $Y(8.65)$ & $\mathrm{Y}(0.58)$ & $\mathrm{Y}(49.71)$ & $\mathrm{Y}(99.11)$ & $\mathrm{Y}(98.84)$ \\
\hline P26 & $\mathrm{Y}(98.59)$ & $\mathrm{Y}(96.65)$ & $\mathrm{Y}(40.88)$ & $\mathrm{Y}(99.52)$ & $\mathrm{Y}(11.30)$ & $\mathrm{Y}(0.54)$ & $\mathrm{Y}(33.95)$ & Y(99.79) & $\mathrm{Y}(99.43)$ \\
\hline P27 & $\mathrm{Y}(98.56)$ & $\mathrm{Y}(96.62)$ & $\mathrm{Y}(41.18)$ & $\mathrm{Y}(99.59)$ & $Y(13.03)$ & $\mathrm{Y}(0.53)$ & $\mathrm{Y}(32.52)$ & $\mathrm{Y}(99.10)$ & $\mathrm{Y}(98.67)$ \\
\hline P28 & $\mathrm{Y}(98.61)$ & Y(96.78) & $\mathrm{Y}(41.16)$ & Y(99.56) & $\mathrm{Y}(13.03)$ & $\mathrm{Y}(0.54)$ & $\mathrm{Y}(32.56)$ & Y(99.77) & $\mathrm{Y}(99.42)$ \\
\hline P29 & $\mathrm{Y}(98.62)$ & $\mathrm{Y}(96.80)$ & $\mathrm{Y}(41.13)$ & $\mathrm{Y}(99.80)$ & $\mathrm{Y}(13.33)$ & $\mathrm{Y}(0.51)$ & $\mathrm{Y}(36.23)$ & $\mathrm{Y}(99.12)$ & $\mathrm{Y}(98.75)$ \\
\hline P30 & $\mathrm{Y}(98.55)$ & $\mathrm{Y}(96.59)$ & $\mathrm{Y}(40.82)$ & $\mathrm{Y}(99.72)$ & $\mathrm{Y}(11.83)$ & $\mathrm{Y}(0.60)$ & $\mathrm{Y}(34.09)$ & $\mathrm{Y}(99.80)$ & $\mathrm{Y}(99.44)$ \\
\hline
\end{tabular}

using a RevertAid Premium Reverse Transcriptase kit from Thermo Fisher Scientific, Inc. Relative quantitative RT-PCR was applied to quantify the mRNAs levels of COL6A1, IL17RC and GAPDH using SYBR-Green Real-Time PCR master mix on the LightCycler480 Real-Time System from Roche (Basel, Switzerland). All experiments were performed in triplicate and normalized to GAPDH.

Statistical analysis. All statistical analyses were performed using SPSS v17.0 software (SPSS, Inc., Chicago, IL, USA). Descriptive data for continuous variables are presented as the mean \pm standard deviation. Student's t-tests were used to determine the age and JOA score differences between patients with or without OPLL gene mutations. The OPLL subtype differences between patients with or without OPLL gene mutations were determined using one-way analysis of variance with a post hoc Fisher's test. $\mathrm{P}<0.05$ was considered to indicate a statistically significant difference.

\section{Results}

WGS analysis. After standard SNP quality control, we performed WGS on 30 DNA samples with an average of 119,583.62 $\mathrm{Mb}$ raw bases from the Illumina Hiseq Xen sequencer. After removing low-quality reads, we obtained an average of 793,363,211 clean reads $(119,004.48 \mathrm{Mb})$. The clean reads of each sample had high Q20 and Q30, indicating high sequencing quality. The average GC content was $41.01 \%$ (Table II). Strict data quality control (QC) was performed during the entire analysis pipeline to obtain accurate data (Table III).

Variant identification. To prioritize potential pathogenic variants, we focused on the identification of rare [multiple allele frequency (MAF) $\leq 0.005$, based on the BGI database] and damaging variants predicted by at least two algorithms (e.g., SIFT, Polyphen-2, MutationTaster, and GERP++). Two 

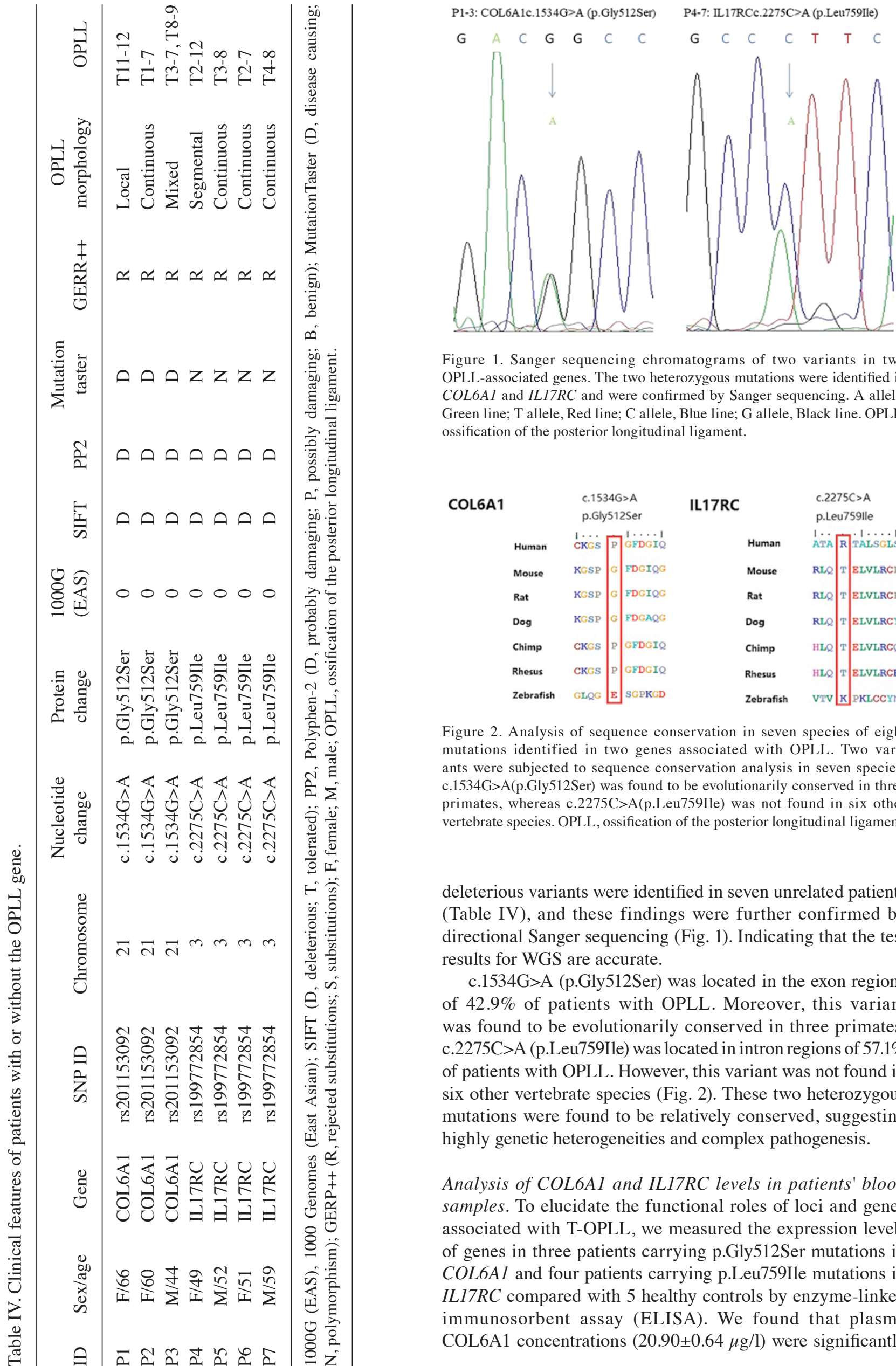

Figure 1. Sanger sequencing chromatograms of two variants in two OPLL-associated genes. The two heterozygous mutations were identified in COL6AI and IL17RC and were confirmed by Sanger sequencing. A allele: Green line; $\mathrm{T}$ allele, Red line; $\mathrm{C}$ allele, Blue line; $\mathrm{G}$ allele, Black line. OPLL, ossification of the posterior longitudinal ligament.

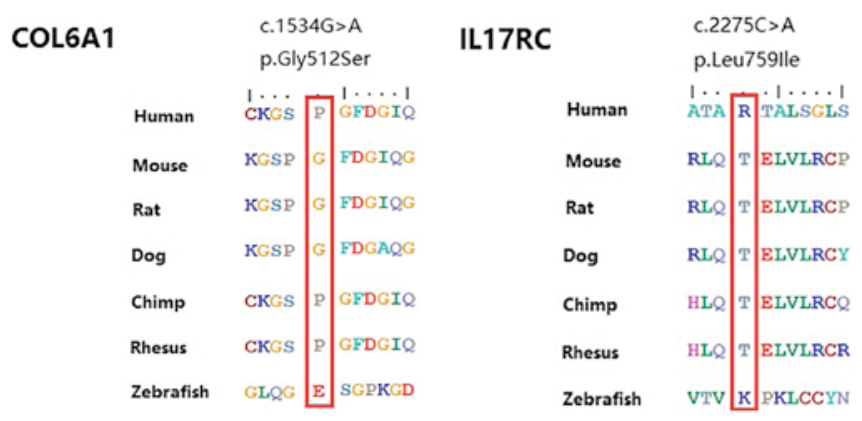

Figure 2. Analysis of sequence conservation in seven species of eight mutations identified in two genes associated with OPLL. Two variants were subjected to sequence conservation analysis in seven species; c.1534G $>$ A(p.Gly512Ser) was found to be evolutionarily conserved in three primates, whereas c.2275C $>\mathrm{A}$ (p.Leu759Ile) was not found in six other vertebrate species. OPLL, ossification of the posterior longitudinal ligament.

deleterious variants were identified in seven unrelated patients (Table IV), and these findings were further confirmed by directional Sanger sequencing (Fig. 1). Indicating that the test results for WGS are accurate.

c.1534G $>$ A (p.Gly512Ser) was located in the exon regions of $42.9 \%$ of patients with OPLL. Moreover, this variant was found to be evolutionarily conserved in three primates. c. $2275 \mathrm{C}>\mathrm{A}$ (p.Leu759Ile) was located in intron regions of $57.1 \%$ of patients with OPLL. However, this variant was not found in six other vertebrate species (Fig. 2). These two heterozygous mutations were found to be relatively conserved, suggesting highly genetic heterogeneities and complex pathogenesis.

Analysis of COL6A1 and IL17RC levels in patients' blood samples. To elucidate the functional roles of loci and genes associated with T-OPLL, we measured the expression levels of genes in three patients carrying p.Gly512Ser mutations in COL6A1 and four patients carrying p.Leu759Ile mutations in IL17RC compared with 5 healthy controls by enzyme-linked immunosorbent assay (ELISA). We found that plasma COL6A1 concentrations $(20.90 \pm 0.64 \mu \mathrm{g} / \mathrm{l})$ were significantly 
Table V. Clinical features of patients with or without OPLL gene mutations.

\begin{tabular}{|c|c|c|c|}
\hline Indices & $\begin{array}{c}\text { Positive } \\
\text { mutation }(n=7)\end{array}$ & $\begin{array}{c}\text { Negative } \\
\text { mutation }(n=23)\end{array}$ & P-value \\
\hline Age (years) & $54.43 \pm 7.56$ & $51.52 \pm 5.57$ & 0.275 \\
\hline Male/female & $3 / 4$ & $10 / 13$ & 0.660 \\
\hline \multicolumn{4}{|c|}{ OPLL subtype (\%) } \\
\hline Continuous & $4(57.1)$ & $3(13)$ & 0.033 \\
\hline Local & $1(14.3)$ & $6(26.1)$ & 0.468 \\
\hline Segmental & $1(14.3)$ & $10(43.4)$ & 0.171 \\
\hline Mixed & $1(14.3)$ & $4(17.4)$ & 0.671 \\
\hline JOA Score & $3.29 \pm 0.95$ & $4.26 \pm 0.45$ & 0.001 \\
\hline
\end{tabular}

OPLL, ossification of the posterior longitudinal ligament; NS, not significant; JOA score, Japanese Orthopedic Association Scoring System for Thoracic Myelopathy (a total of 11 points).
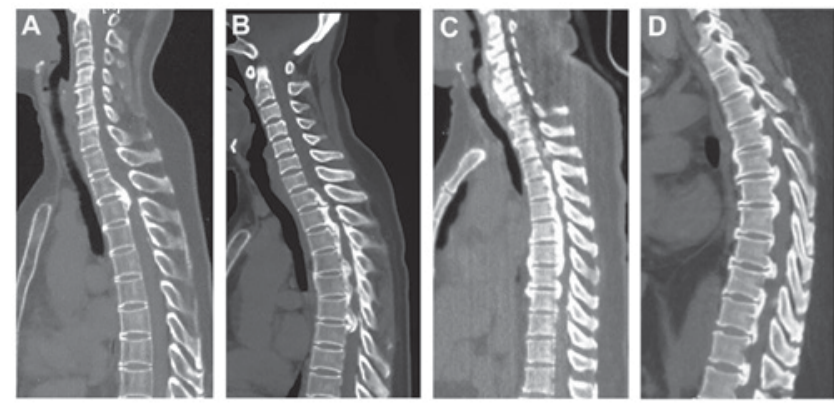

Figure 3.2D-CT showed that four patients had deleterious variants. (A) The Local subtype of T-OPLL. (B) The Mixed subtype of T-OPLL. (C) The Segmental subtype of T-OPLL. (D) The Continuous subtype of T-OPLL. 2D-CT, two-dimensional computed tomography; T-OPLL, thoracic ossification of the posterior longitudinal ligament.

higher $(\mathrm{P}<0.05)$, approximately 4-fold higher than those in healthy controls $(4.79 \pm 1.18 \mu \mathrm{g} / \mathrm{l})$. Moreover, plasma IL17RC concentrations $(6.13 \pm 0.36 \mu \mathrm{g} / \mathrm{l})$ were also significantly higher $(\mathrm{P}<0.05)$, approximately 2 -fold higher than those in healthy controls $(2.89 \pm 0.30 \mu \mathrm{g} / \mathrm{l})$. We performed RT-qPCR analysis using peripheral blood cells and found that COL6A1 mRNA levels in three patients carrying the p.Gly512Ser mutation were approximately 6-fold higher than those in healthy controls, the difference was statistically significant $(\mathrm{P}<0.05)$. The IL17RC mRNA levels in four patients carrying the p.Leu759Ile mutation were approximately 4-fold higher than those in the healthy controls, the difference was also statistically significant $(\mathrm{P}<0.05)$. Compared with healthy controls group, we observed that these two mutations significantly increase their respective gene expressed, suggesting that these two potential pathogenic loci have potential effect on their respective gene expressed cells.

Genotype-phenotype analysis.Phenotype-genotype correlations were analysed among the seven patients with missense mutations and 23 patients without significant mutations (Table V). No differences were found between these two groups in terms of
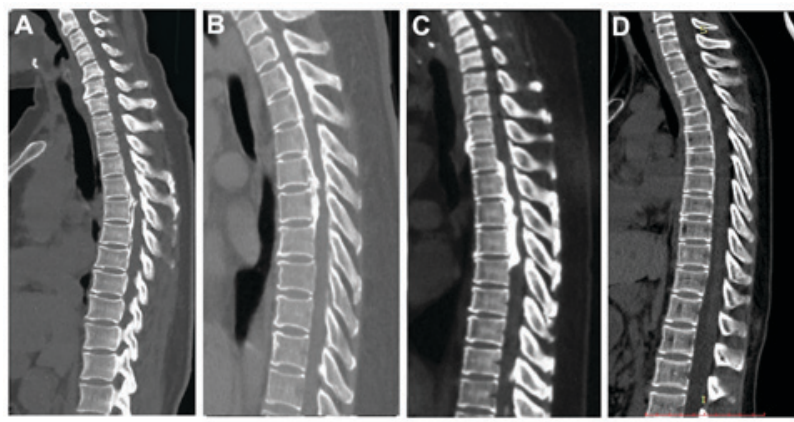

Figure 4. 2D-CT showed that three patients had deleterious variants and one healthy control. (A-C) The Continuous subtype of T-OPLL. (D) A healthy control. 2D-CT, two-dimensional computed tomography; T-OPLL, thoracic ossification of the posterior longitudinal ligament.

sex or age at diagnosis. Two-dimensional computed tomography (2D-CT) scans of seven patients with OPLL harbouring deleterious variants and one healthy control are shown in Figs. 3 and 4. More specifically, one patient belonged to the Local subtype (Fig. 3A), one patient belonged to the Mixed subtype (Fig. 3B), one patient showed the Segmental subtype (Fig. 3C), and four patients showed the Continuous subtype (Figs. 3D and 4A-C), Fig. 4D showed the healthy control. However, the JOA score for thoracic myelopathy was significantly lower in patients with rare missense mutations compared with patients without mutations $(\mathrm{P}=0.001)$. Additionally, radiological analysis of OPLL morphology (17) revealed that the frequency of the continuous subtype was significantly higher in patients with rare missense mutations than in patients without mutations $(\mathrm{P}=0.033)$. Moreover, four mutation-positive patients (57.1\%) showed the continuous subtype, one patient (14.3\%) showed the local subtype, one patient (14.3\%) showed the segmental subtype, and one patient (14.3\%) showed the mixed subtype. By contrast, three (13\%) mutation-negative patients showed the continuous subtype, six patients $(26.1 \%)$ showed the local subtype, 10 patients (43.4\%) showed the segmental subtype, and four patients (17.4\%) without mutations showed the mixed subtype.

\section{Discussion}

COL6A1, which encodes the $\alpha 1$ chain of type VI collagen, is located on chromosome $21 \mathrm{q} 22.3$ and spans approximately $23.3 \mathrm{~kb}$. Additionally, as a major structural component of microfibrils, COL6A1 plays a role in maintaining the integrity of various tissues and has been shown to be associated with OPLL in Japanese and Chinese populations $(8,9)$. Previously, COL6A1 mutations associated with OPLL were found in intronic regions. However, in the present study, we report for the first time a mutation, c.1534G>A(p.Gly512Ser)/COL6A1, in the exonic region that is associated with T-OPLL. This mutation may be involved in transcriptional regulation, and analysis using the SIFT, Polyphen-2, and MutationTaster algorithms suggested that this mutation may have an adverse impact on the structure and function of the encoded protein. Additionally, COL6A1 may serve as a scaffold for osteoblastic or pre-osteoblastic cells or chondrocytes that subsequently undergo membranous or endochondral ossification (26). Therefore, the molecular variants of extracellular proteins may 
be involved in the ectopic bone formation that is observed in patients with OPLL (27).

The IL17RC gene encodes a single-pass type I transmembrane protein located on chromosome region 3 p25.3 to 3p24.1, spanning approximately $16,550 \mathrm{bp}$ (28). OPLL results in increased bone formation in ligament tissue, and there is some evidence showing a correlation between OPLL and increased systemic bone mineral density. ILI7RC accelerates osteoblast differentiation; a recent study indicated that dysfunction of both IL17RC and the IL-17 cytokine/IL-17R signalling axis is indispensable for osteoblastogenesis (29). Moreover, the TGF- $\beta$ signalling pathway is known to be associated with the formation of bone mass and matrix (30). The IL17RC gene may also be involved in bone metabolism through canonical TGF- $\beta$ signalling (31). However, this variant was not found in six other vertebrate species, suggesting high genetic heterogeneities. In this study, we identified c.2275C >A(p.Leu759Ile)/IL17RC, which had not previously been reported to be associated with OPLL, using the standard potential pathogenic variant method, thereby linking ILI7RC to OPLL and suggesting that this mutation, as well as the mutation in COL6A1, may be involved in bone development.

At present, several lines of evidence suggest that OPLL seems to occur and develop as a result of systemic and local factors in combination with a genetic abnormality (32-34). The progression of the disease also affects the gene expression in peripheral blood cells, several genes are highly expressed in peripheral blood of patients with OPLL $(7,32)$. In addition, peripheral blood is easily accessible and routinely used for diagnostic laboratory analysis and thus is a good resource for additional tests that might define extent of T-OPLL. Therefore, what we observed an increased level of COL6A1 and IL17RC with two heterozygous mutations in the patient's peripheral blood revealed its potential role in the pathogenicity of T-OPLL.

There was a limitation in the present study. The sample size is small, larger scale studies is necessary. However, the T-OPLL is primarily found in individuals in northeast Asia, the prevalence of T-OPLL in Japanese individuals is only $0.8-1.6 \%(6,35)$, the prevalence of this disease is very rare. To the best of our knowledge, our 30 sample size is the largest cases of single center. To accomplish this issue, international collaboration is the good way to go.

In summary, in this study, we identified mutations in Han Chinese patients with T-OPLL for the first time using WGS. From our analysis, we found two new potential pathogenic loci for OPLL: c.1534G>A(p.Gly512Ser) in the COL6AI gene, which has previously been reported to be associated with OPLL, and c.2275C >A(p.Leu759Ile) in the IL17RC gene, which had not previously been reported to be associated with OPLL. The results of the current study provide insights into the molecular aetiology of OPLL. Further genetic and functional studies, including studies with more participants of other ethnicities, are needed to confirm these positive findings.

\section{Acknowledgements}

This study was supported by the National Natural Science Foundation of China (no. 81472041). Thanks are due to Medical Research Center of Peking University Third Hospital for providing technical guidance.

\section{References}

1. Kim KH, Kuh SU, Park JY, Lee SJ, Park HS, Chin DK, Kim KS and Cho YE: Association between BMP-2 and COL6A1 gene polymorphisms with susceptibility to ossification of the posterior longitudinal ligament of the cervical spine in Korean patients and family members. Genet Mol Res 13: 2240-2247, 2014.

2. Nagashima C: Cervical myelopathy due to ossification of the posterior longitudinal ligament. J Neurosurg 37: 653-660, 1972.

3. Yonemori K, Imamura T, Ishidou Y, Okano T, Matsunaga S, Yoshida H, Kato M, Sampath TK, Miyazono K, ten Dijke P and Sakou T: Bone morphogenetic protein receptors and activin receptors are highly expressed in ossified ligament tissues of patients with ossification of the posterior longitudinal ligament. Am J Pathol 150: 1335-1347, 1997.

4. Ikegawa S: Genetics of ossification of the posterior longitudinal ligament of the spine: A mini review. J Bone Meta 21: 127-132, 2014.

5. Tsuji T, Chiba K, Hosogane N, Fujita N, Hikata T, Iwanami A, Watanabe K, Ishii K, Toyama Y, Nakamura M and Matsumoto M: Epidemiological survey of ossification of the posterior longitudinal ligament by using clinical investigation registration forms. J Orthop Sci 21: 291-294, 2016.

6. Fujimori T, Watabe T, Iwamoto Y, Hamada S, Iwasaki M and Oda T: Prevalence, concomitance and distribution of ossification of the spinal ligaments: Results of Whole Spine CT Scans in 1500 Japanese Patients. Spine (Phila Pa 1976) 41: 1668-1676, 2016.

7. Chen X, Guo J, Cai T, Zhang F, Pan S, Zhang L, Wang S, Zhou F, Diao Y, Zhao Y, et al: Targeted next-generation sequencing reveals multiple deleterious variants in OPLL-associated genes. Sci Rep 6: 26962, 2016.

8. Kong Q, Ma X, Li F, Guo Z, Qi Q, Li W, Yuan H, Wang Z and Chen Z: COL6A1 polymorphisms associated with ossification of the ligamentum flavum and ossification of the posterior longitudinal ligament. Spine (Phila Pa 1976) 32: 2834-2838, 2007.

9. Tanaka T, Ikari K, Furushima K, Okada A, Tanaka H, Furukawa K, Yoshida K, Ikeda T, Ikegawa S, Hunt SC, et al: Genomewide linkage and linkage disequilibrium analyses identify COL6A1, on chromosome 21, as the locus for ossification of the posterior longitudinal ligament of the spine. Am J Hum Genet 73: 812-822, 2003.

10. Koga H, Sakou T, Taketomi E, Hayashi K, Numasawa T, Harata S, Yone K, Matsunaga S, Otterud B, Inoue I and Leppert M: Genetic mapping of ossification of the posterior longitudinal ligament of the spine. Am J Hum Genet 62: 1460-1467, 1998.

11. Wang H, Liu D, Yang Z, Tian B, Li J, Meng X, Wang Z, Yang H and Lin X: Association of bone morphogenetic protein-2 gene polymorphisms with susceptibility to ossification of the posterior longitudinal ligament of the spine and its severity in Chinese patients. Eur Spine J 17: 956-964, 2008.

12. Kamiya M, Harada A, Mizuno M, Iwata H and Yamada Y: Association between a polymorphism of the transforming growth factor-beta1 gene and genetic susceptibility to ossification of the posterior longitudinal ligament in Japanese patients. Spine (Phila Pa 1976) 26: 1264-1267, 2001.

13. Ogata N, Koshizuka Y, Miura T, Iwasaki M, Hosoi T, Shiraki M, Seichi A, Nakamura K and Kawaguchi H: Association of bone metabolism regulatory factor gene polymorphisms with susceptibility to ossification of the posterior longitudinal ligament of the spine and its severity. Spine (Phila Pa 1976) 27: 1765-1771, 2002.

14. Kim DH, Jeong YS, Chon J, Yoo SD, Kim HS, Kang SW, Chung JH, Kim KT and Yun DH: Association between interleukin 15 receptor, alpha (IL15RA) polymorphism and Korean patients with ossification of the posterior longitudinal ligament. Cytokine 55: 343-346, 2011.

15. Guo Q, Lv SZ, Wu SW, Tian X and Li ZY: Association between single nucleotide polymorphism of IL15RA gene with susceptibility to ossification of the posterior longitudinal ligament of the spine. J Orthop Surg Res 9: 103, 2014.

16. Liu Y, Zhao Y, Chen Y, Shi G and Yuan W: RUNX2 polymorphisms associated with OPLL and OLF in the Han population. Clin Orthop Relat Res 468: 3333-3341, 2010.

17. Tsuyama N: Ossification of the posterior longitudinal ligament of the spine. Clin Orthop Relat Res: 71-84, 1984.

18. Li H and Durbin R: Fast and accurate long-read alignment with Burrows-Wheeler transform. Bioinformatics 26: 589-595, 2010. 
19. Li H and Durbin R: Fast and accurate short read alignment with Burrows-Wheeler transform. Bioinformatics 25: 1754-1760, 2009.

20. McKenna A, Hanna M, Banks E, Sivachenko A, Cibulskis K, Kernytsky A, Garimella K, Altshuler D, Gabriel S, Daly M and DePristo MA: The genome analysis toolkit: A MapReduce framework for analyzing next-generation DNA sequencing data. Genome Res 20: 1297-1303, 2010.

21. DePristo MA, Banks E, Poplin R, Garimella KV, Maguire JR, Hartl C, Philippakis AA, del Angel G, Rivas MA, Hanna M, et al: A framework for variation discovery and genotyping using next-generation DNA sequencing data. Nat Genet 43: 491-498, 2011.

22. Sim NL, Kumar P, Hu J, Henikoff S, Schneider G and Ng PC: SIFT web server: Predicting effects of amino acid substitutions on proteins. Nucleic Acids Res 40: W452-W457, 2012.

23. Adzhubei IA, Schmidt S, Peshkin L, Ramensky VE, Gerasimova A, Bork P, Kondrashov AS and Sunyaev SR: A method and server for predicting damaging missense mutations. Nat Methods 7: 248-249, 2010.

24. Schwarz JM, Cooper DN, Schuelke M and Seelow D: MutationTaster2: Mutation prediction for the deep-sequencing age. Nat Methods 11: 361-362, 2014.

25. Davydov EV, Goode DL, Sirota M, Cooper GM, Sidow A and Batzoglou S: Identifying a high fraction of the human genome to be under selective constraint using GERP++. PLoS Comput Biol 6: e1001025, 2010.

26. Wiberg C, Klatt AR, Wagener R, Paulsson M, Bateman JF, Heinegård D and Mörgelin M: Complexes of matrilin-1 and biglycan or decorin connect collagen VI microfibrils to both collagen II and aggrecan. J Biol Chem 278: 37698-37704, 2003.

27. Tsukahara S, Miyazawa N, Akagawa H, Forejtova S, Pavelka K, Tanaka T, Toh S, Tajima A, Akiyama I and Inoue I: COL6A1, the candidate gene for ossification of the posterior longitudinal ligament, is associated with diffuse idiopathic skeletal hyperostosis in Japanese. Spine (Phila Pa 1976) 30: 2321-2324, 2005.
28. Ho AW and Gaffen SL: IL-17RC: A partner in IL-17 signaling and beyond. Semin Immunopathol 32: 33-42, 2010.

29. Huang H, Kim HJ, Chang EJ, Lee ZH, Hwang SJ, Kim HM, Lee Y and Kim HH: IL-17 stimulates the proliferation and differentiation of human mesenchymal stem cells: Implications for bone remodeling. Cell Death Differ 16: 1332-1343, 2009.

30. Mohammad KS, Chen CG, Balooch G, Stebbins E, McKenna CR, Davis H, Niewolna M, Peng XH, Nguyen DH, Ionova-Martin SS, et al: Pharmacologic inhibition of the TGF-beta type I receptor kinase has anabolic and anti-catabolic effects on bone. PLoS One 4: e5275, 2009.

31. Mukherjee S, Schaller MA, Neupane R, Kunkel SL and Lukacs NW: Regulation of T cell activation by Notch ligand, DLL4, promotes IL-17 production and Rorc activation. J Immunol 182: 7381-7388, 2009.

32. Niu CC, Lin SS, Yuan LJ, Chen LH, Yang CY, Chung AN, Lu ML, Tsai TT, Lai PL and Chen WJ: Correlation of blood bone turnover biomarkers and Wnt signaling antagonists with AS, DISH, OPLL, and OYL. BMC Musculoskelet Disord 18: 61, 2017.

33. Ikegawa S: Genomic study of ossification of the posterior longitudinal ligament of the spine. Proc Jpn Acad Ser B Phys Biol Sci 90: 405-412, 2014.

34. Nakajima M, Takahashi A, Tsuji T, Karasugi T, Baba H, Uchida K, Kawabata S, Okawa A, Shindo S, Takeuchi K, et al: A genome-wide association study identifies susceptibility loci for ossification of the posterior longitudinal ligament of the spine. Nat Genet 46: 1012-1016, 2014.

35. Ohtsuka K, Terayama K, Yanagihara M, Wada K, Kasuga K, Machida T and Matsushima S: A radiological population study on the ossification of the posterior longitudinal ligament in the spine. Arch Orthop Trauma Surg 106: 89-93, 1987. 